\title{
Prevalence of local allergic rhinitis to Dermatophagoides pteronyssinus in chronic rhinitis with negative skin prick test
}

\author{
Pongsakorn Tantilipikorn, ${ }^{1}$ Panlop Siriboonkoom, ${ }^{1}$ Nitat Sookrung, ${ }^{2}$ Arathaya Thianboonsong, ${ }^{1}$ Triphoom Suwanwech, ${ }^{1}$ \\ Bangon Pinkaew, ${ }^{1}$ Paraya Asanasaen ${ }^{1}$
}

\begin{abstract}
Background: Local allergic rhinitis (LAR) is a localized nasal allergic response in the absence of systemic atopy that is characterized by local production of specific immunoglobulin E (sIgE), and a positive response to NAPT (nasal allergen provocation test).
\end{abstract}

Objective: The objective of this study is to investigate the prevalence of LAR in adults with chronic rhinitis (CR) and negative skin prick test to Dermatophagoides pteronyssinus (Dp), and to assess the clinical characteristics of LAR, comparing to non-allergic rhinitis (NAR).

Methods: Patients with history of CR with negative skin prick test (SPT) to Dp were recruited during January 2015-April 2016. Demographic and clinical data were obtained, and then a NAPT with Dp was performed. The immediate response to NAPT-Dp was assessed using clinical symptom score, visual analogue scale, peak nasal inspiratory flow, and acoustic rhinometry. Nasal lavage was evaluated for nasal sIgE and tryptase level.

Results: Sixty-two CR patients were recruited. NAPT-Dp was positive in 15/62 (24.2\%) of CR patients. Most LAR patients were female (73.3\%), and the mean age of all patients was $36.1 \pm 10.4$ years. Our evaluation of patient characteristics revealed sneezing to be the only evaluated factor to be statistically significantly different between groups [odds ratio (OR): 7.75, 95\% confidence interval (CI): 1.91-31.48; $p=0.002]$.

Conclusions: The prevalence of LAR to Dp in Thai adults with CR and negative skin prick test was 24.2\%. Most LAR patients had moderate-severe persistent severity. The clinical characteristics of LAR, sneezing was shown to be a significantly dominant symptom in LAR than in NAR.

Key words: Local allergic rhinitis, allergic rhinitis, chronic rhinitis, nasal allergen provocation test, nasal challenge test

From:

${ }^{1}$ Division of Rhinology \& Allergy, Department of

Otorhinolaryngology, Faculty of Medicine Siriraj Hospital, Mahidol University, Bangkok, Thailand

${ }^{2}$ Office of Research and Development, Faculty of Medicine, Siriraj Hospital, Mahidol University, Bangkok, Thailand

\section{Corresponding author:}

Pongsakorn Tantilipikorn

Division of Rhinology \& Allergy, Department of Otorhinolaryngology

Faculty of Medicine Siriraj Hospital, Mahidol University

2 Wanglang Road, Bangkoknoi

Bangkok 10700, Thailand

E-mail: pongsakorn.tan@mahidol.ac.th

\section{Introduction}

Rhinitis is defined as inflammation of the nasal epithelium, and is diagnosed in the simultaneous presence of at least two of the following nasal symptoms: rhinorrhea, nasal blockage, nasal itching, and sneezing. ${ }^{1,2}$ Rhinitis adversely impacts patient physical, mental, and social quality of life,,$^{3-5}$ and it is often associated with asthma, rhinosinusitis, and conjunctivitis. The high prevalence of and the comorbidities associated with rhinitis make it a healthcare burden in many societies. ${ }^{6}$ Chronic rhinitis is generally classified as allergic rhinitis (AR) or nonallergic rhinitis (NAR) based on clinical history and skin prick test (SPT) or serum specific $\operatorname{IgE}(\mathrm{s} \operatorname{IgE}){ }^{3}$ In Thailand, the prevalence of AR and NAR in CR was reported to be $20 \%-40 \%$ and $60 \%-80 \%$, respectively. ${ }^{7-10}$ 
In 1975, Huggins and Brostoff ${ }^{11}$ studied patients diagnosed with clinical AR who had negative SPT, but who had positive nasal allergen provocation test (NAPT) and nasal sIgE after being exposed to aeroallergen. Later, Rondon, et al. ${ }^{12}$ introduced the concept of localized mucosal allergic disease in the absence of systemic atopy, which is now referred to as local allergic rhinitis (LAR) or entopy. LAR is characterized by local production of sIgE antibodies, nasal Th2 response during natural exposure to aeroallergens, and a positive response to NAPT. Levels of sIgE, tryptase, and eosinophil cationic protein (ECP) were found to be increased in the secretions of LAR patients. ${ }^{13}$

Even though AR and LAR are considered different entities, they share some similarities, especially relative to management. Medications, such as oral antihistamines and intranasal steroids, were reported to have benefit in LAR. Moreover, a randomized, double-blind, placebo controlled phase 2 clinical trial to investigate the efficacy and safety of subcutaneous immunotherapy (SCIT) with Dermatophagoides pteronyssinus (Dp) in patients with LAR reported a favorable outcome. ${ }^{14,15}$

Previous studies from the European Union and the United States reported prevalence rates of LAR in NAR that ranged from $47 \%$ to $62.5 \%{ }^{12,16}$ The prevalence of LAR was reported to be about $7-19 \%$ in China, and $9-10 \%$ in Korea. ${ }^{17,18}$ In Thailand, the prevalence of LAR in pediatric patients is $3.7 \%{ }^{19}$ Until recently, there was no study about LAR in adult patients in Thailand.

The aim of this study was to investigate the prevalence of LAR to Dp in adults with CR and negative skin prick test, and to assess the clinical characteristics and severity of LAR in Thailand.

\section{Materials and Methods Study population}

This cross-sectional study included patients aged 18-70 years with history of chronic rhinitis longer than 1 year that were attending the outpatient allergy clinic of the Department of Otorhinolaryngology, Faculty of Medicine Siriraj Hospital, Mahidol University, Bangkok, Thailand during the January 2015 to April 2016 study period. The approval protocol number (EC1) from Siriraj Institutional Review Board (SIRB) is COA no.660/2557, and written informed consent was obtained from all patients. This study complied with the principles set forth in the Declaration of Helsinki (1964) and all of its subsequent amendments.

Patients that satisfied both of the following inclusion criteria were included: 1) diagnosed with CR by negative SPT; and, 2) discontinued use of the following medications for the following specified period of time prior to the start of the study: systemic corticosteroid (4 weeks), intranasal corticosteroid ( 2 weeks), oral antihistamine (1 week), and topical nasal decongestant (1 day prior). Patients having one or more of the following were excluded: 1) underlying diseases, including chronic rhinosinusitis with or without nasal polyp, severe deviated nasal septum, immunological diseases, chronic renal disease, hepatobiliary disease, cardiovascular disease, or cancer; 2) uncontrolled asthma by predicted peak expiratory flow rate $(\mathrm{PEF})<70 \%$; 3 ) recent history of respiratory tract infection within the previous weeks; 4) pregnancy or lactation; and/or, 5) nasal hyperreactivity.

Patient baseline characteristics were recorded, including age, gender, disease duration, location of residence (urban or rural), family history of atopic diseases, and precipitating factors. The subjective evaluations were total symptom score (TSS) and visual analog scale (VAS), and the objective evaluations were acoustic rhinometer (ARM) and peak nasal inspiratory flow (PNIF). Patients underwent nasal allergen provocation test (NAPT) for Dp, with nasal lavage performed 30 minutes prior to the test. For the nasal hypersensitivity test, patients were challenged with diluent consisting of phosphate buffered saline with $0.4 \%$ phenol. Patients found to be positive were excluded. NAPT was performed using an initial Dp concentration of $200 \mathrm{AU}$, with increases to $500 \mathrm{AU}$ and 1000 AU until the test was positive. Fifteen minutes after each nasal provocation test, patients were evaluated for TSS, VAS, ARM, and PNIF. At 60 minutes after the test, nasal lavage was performed, patient TSS, VAS, ARM, and PNIF were recorded, and nasal lavage was measured for sIgE and tryptase.

\section{Definitions}

Rhinitis symptoms were classified according to the Allergic Rhinitis and its Impact on Asthma (ARIA) guidelines. ${ }^{20}$ Rhinitis was classified as intermittent (symptoms present $<4$ days/week or for $<4$ consecutive weeks) or persistent (symptoms present $>4$ days/week and for $>4$ consecutive weeks) (4). Regarding rhinitis severity, the number of impaired items, including sleep, daily activities/sport/leisure, work/school performance, and troublesome symptoms, was used to categorize rhinitis as mild (no affected items) or moderate-to-severe (at least one affected item).

\section{Skin prick test (SPT)}

SPT was performed using a panel of the most prevalent local aeroallergens, including house dust mites (Dermatophagoides pteronyssinus), American and German cockroaches, cat and dog dander, grass pollens (Bermuda and Johnson), acacia, careless weeds, and molds (Alternaria spp., Cladosporium spp., Penicillium spp., Aspergillus spp., and Fusarium spp.). Commercial allergens were purchased from ALK Abello (Port Washington, NY, USA). Histamine $(10 \mathrm{mg} / \mathrm{ml})$ and saline were used as positive and negative control, respectively. A positive SPT was defined as a wheal diameter of $\geq 3 \mathrm{~mm}$ compared to the negative control, to $\geq 1$ aeroallergen. Study patients were instructed to discontinue antihistamine for at least 7 days before SPT.

\section{Nasal allergen provocation test (NAPT)}

Standard anterior rhinoscopy was performed to exclude patients with pre-existing condition within the nose. A challenge test to phosphate-buffered saline (PBS) was performed to exclude any non-specific nasal hyper-reactivity. If patients had no reaction to PBS, NAPT with Dp (NAPT-Dp) was performed using Dp extract solutions of 200, 600, and $2000 \mathrm{AU} /$ $\mathrm{ml}$ at 15 -minute intervals. Two puffs $(0.125 \mathrm{ml}$ each $)$ of solution were introduced into each nasal passage using a metered nasal spray pump. The immediate response to NAPT-Dp was 
assessed using clinical symptom score, peak nasal inspiratory flow (PNIF), and acoustic rhinometry (ARM).

\section{The symptom score}

The symptom score was based primarily on the following symptoms: nasal obstruction [breathing difficulty (1 point), unilateral nasal obstruction (2 points), bilateral nasal obstruction (3 points)], rhinorrhea [anterior (1 point), posterior ( 1 point)], itchiness [nose ( 1 point), ear or palate ( 1 point)], sneezing [0-2 times ( 0 points), 3-4 times (1 point), $\geq 5$ times (3 points)], and ocular symptoms [1 point]. The maximum possible score was 11 points. ${ }^{21}$ Severity of nasal symptoms was also assessed using visual analog scale (VAS) (range: 0-10).

Nasal airflow was assessed using a peak nasal inspiratory flow (PNIF) meter (In-Check Inspiratory Flow Meter with face mask; Clement Clarke International, Harlow, UK). The highest PNIF value from three measurements was used for calculation. ${ }^{21,22}$ Nasal patency was assessed by Eccovision Acoustic Rhinometer (Model AR-1003; Hood Laboratories; Pembroke, MA, USA) following the guidelines set forth by the Standardization Committee on Acoustic Rhinometry. The minimum cross-sectional area (MCA) of each nasal cavity was measured separately, and the mean MCA of the two cavities was calculated. ${ }^{23}$

NAPT was considered positive when a nasal obstruction was observed that satisfied one or the other of the follow criteria: (1) the ARM showed a reduction of $>25 \%$ of the MCA of the nasal cavity, or (2) the nasal airflow decreased $>40 \%$ of the baseline value - both regardless of the clinical symptom score. If nasal airflow decreases by $>20 \%$ of the baseline value, it must be combined with a symptom score greater than $5 .^{21,22}$

Nasal lavage and sample processing were performed according to the following procedures. Study patients angled their necks up approximately $30^{\circ}$ from horizontal while in a sitting position. Patients were advised not to breathe or swallow, and then $3 \mathrm{~mL}$ of normal saline was instilled into each nostril. After 10 seconds, subjects angled their necks down to a negative $30^{\circ}$ from horizontal and expelled the mixture of mucus and saline into a bowl, which was stored on ice until the end of the NAPT. Specimens were maintained at $4^{\circ} \mathrm{C}$ until they were centrifuged at 2,000 rpm $(1,069 \mathrm{~g})$ for $7 \mathrm{~min}$ at $4^{\circ} \mathrm{C}$. The supernatant was stored at $20^{\circ} \mathrm{C}$ until nasal tryptase and sIgE-Dp were measured. Total tryptase and sIgE-Dp levels were measured by UniCAP Tryptase Immunoassay (Pharmacia, Uppsala, Sweden) according to the manufacturer's instructions. ${ }^{11,24}$

\section{Statistical analysis}

Data analysis was performed using SPSS Statistics for Windows version 18.0 (SPSS, Inc., Chicago IL, USA). Categorical data are presented as number and percentage, and continuous data are given as mean \pm standard deviation (SD) for parametric variables and median and interquartile range (IQR) for nonparametric variables. Clinical data were compared between LAR and NAR using chi-square test. Logistic regression analysis was used to identify factors significantly associated with positive NAPT. A $p$-value less than 0.05 was considered statistically significant.

\section{Results}

Sixty-two CR patients were included. Baseline demographic and clinical characteristics are shown in Table 1. The mean age was $42.4 \pm 13.0$ years, the median disease duration was 5 years (IQR: $2-10$ ), and $72.6 \%$ of patients were female.

Table 1. Baseline demographic and clinical characteristics $(\mathrm{N}=62)$

$\begin{array}{lc}\text { Characteristics } & \text { Values } \\ \text { Age (years), mean } \pm \text { SD } & 42.4 \pm 13.0 \\ \text { Gender, n (\%) } & \\ \text { Female } & 45(72.6 \%) \\ \text { Male } & 17(27.4 \%) \\ \text { Disease duration (years), median (IQR) } & 5(2,10) \\ \text { Location of residence, } n(\%) & \\ \text { Urban } & 40(64.5 \%) \\ \text { Rural } & 22(35.5 \%) \\ \text { Family history of atopic diseases, n (\%) } & 27(43.5 \%) \\ \text { Precipitating factors, } n \text { (\%) } & \\ \text { Dog } & 19(30.6 \%) \\ \text { Cat } & 14(22.6 \%) \\ \text { Smoke } & 5(8.1 \%) \\ \text { Chemical agent } & 20(32.3 \%)\end{array}$

Abbreviations: SD, standard deviation; IQR, interquartile range

Fifteen patients (24.2\%) were diagnosed with LAR by positive NAPT to Dp. TSS, VAS, MCA, and PNIF results were all significantly different between before and after positive NAPT (all $p<0.001$ ) (Figure 1).

Although patients in the LAR group tended to be younger, no significant difference was observed for age, gender, disease duration, or family history of atopic diseases between the LAR and NAR groups (Table 2). With regard to our evaluation of nasal symptoms, only sneezing was found to be statistically significantly different between groups [odds ratio (OR): 7.75, 95\% confidence interval (CI): 1.91-31.48; $p=0.002$ ].

We evaluated nasal lavage from LAR patients at 1 hour after positive NAPT. Using a positive cutoff value of $\geq 0.35$ $\mathrm{kUA} / \mathrm{L}$ for $\mathrm{sIgE}$ and $>11.5 \mathrm{ng} / \mathrm{ml}$ for tryptase, our analysis revealed no patients positive for nasal sIgE, and 1 patient positive for nasal tryptase. 

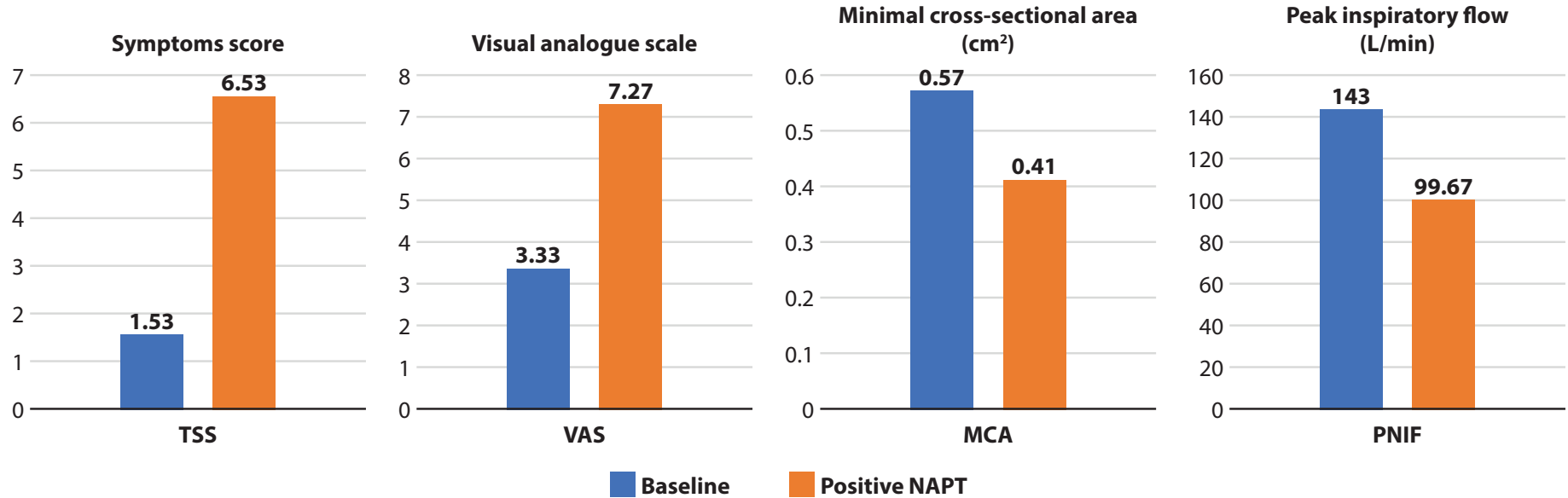

Figure 1. Clinical parameters of local allergic rhinitis patients

Abbreviations: VAS, visual analog scale; MCA, minimal cross-sectional area; PNIF, peak inspiratory flow; NAPT, nasal allergen provocation test

Table 2. Clinical characteristics compared between LAR and NAR patients

\begin{tabular}{|c|c|c|c|c|c|}
\hline Characteristics & $\operatorname{LAR}(\mathbf{n}=15)$ & $\operatorname{NAR}(n=47)$ & Odds ratio (OR) & $95 \% \mathrm{CI}$ & $p$ \\
\hline Number, n (\%) & $15(24.2 \%)$ & $47(75.8 \%)$ & & & \\
\hline \multicolumn{6}{|l|}{ Age (years), n (\%) } \\
\hline $18-35$ & $8(53.3 \%)$ & $14(29.8 \%)$ & 0.37 & $0.11-1.22$ & 0.097 \\
\hline$>35$ & $7(46.7 \%)$ & $33(70.2 \%)$ & & & \\
\hline \multicolumn{6}{|l|}{ Gender, n (\%) } \\
\hline Female & $11(73.3 \%)$ & $34(72.3 \%)$ & 1.05 & $0.28-3.90$ & 1.000 \\
\hline Male & $4(26.7 \%)$ & $13(27.7 \%)$ & & & \\
\hline Disease duration (years), median (IQR) & $5(2,10)$ & $5(2,10)$ & & & \\
\hline Family history of atopic diseases, n (\%) & $5(33.3 \%)$ & $22(46.8 \%)$ & 0.74 & $0.21-2.50$ & 0.359 \\
\hline \multicolumn{6}{|l|}{ Nasal symptoms, n (\%) } \\
\hline Nasal itching & $8(53.3 \%)$ & $18(38.3 \%)$ & 1.84 & $0.57-5.95$ & 0.304 \\
\hline Sneezing & $12(80.0 \%)$ & $16(34.0 \%)$ & 7.75 & $1.91-31.48$ & 0.002 \\
\hline Nasal blockage & $11(73.3 \%)$ & $35(74.5 \%)$ & 0.94 & $0.25-3.53$ & 1.000 \\
\hline Rhinorrhea & $10(66.7 \%)$ & 27 (58.7\%) & 1.48 & $0.44-5.02$ & 0.583 \\
\hline
\end{tabular}

A $p$-value $<0.05$ indicates statistical significance

Abbreviations: LAR, local allergic rhinitis; NAR, non-allergic rhinitis; CI confidence interval; IQR, interquartile range

\section{Discussion}

The aim of this study was to investigate the prevalence of LAR to Dp in adults with CR and negative skin prick test, and to assess the clinical characteristics and severity of LAR to $\mathrm{Dp}$ in Thailand. This is the first study to examine LAR to Dp in Thai adults. The findings of this study will enhance our knowledge about LAR and our ability to manage patients with this condition, and these enhancements will result in improved patient outcomes.

In this study, the prevalence of LAR to Dp was $24.2 \%$. Compared to other studies, that proportion was higher than the rate found in Thai children (3.7\%) and in other Asian countries (China 19.0\%, Korean 10.8\%), but lower than in Western populations (47-62\%). This disparity in prevalence may be explained by differences in environment, genetic factors and the duration of withheld intranasal steroid before NAPT. Other factors that may affect differences in reported prevalence include the diagnostic criteria (NAPT or nasal sIgE) and the number of allergens used in the testing process.

Relative to clinical characteristics, we found LAR patients to be younger than NAR patients, female predominance, and no difference between groups for disease duration or family history of atopic diseases (all $p>0.05$ ). A majority of patients had moderate to severe persistent symptoms, which is not different from previous studies. ${ }^{25}$ Of all of the evaluated clinical characteristics, sneezing was the only factor to be significantly different between LAR and NAR ( $80 \%$ vs. $34 \%$; $p=0.002)$. 
Regarding our evaluation for nasal sIgE and tryptase after positive NAPT, no patients were positive for sIgE, and only 1 patient was found to be positive for tryptase. Consistent with our finding, some previous studies found that an increase in nasal sIgE or tryptase was not demonstrated in all LAR patients. A study in Thai children by Buntarickpornpan, et al. revealed no sIgE or tryptase positive results. ${ }^{19}$ Rondon, et al. reported only $22 \%$ of patients with positive nasal sIgE, while Lopez, et al. reported increased nasal $\operatorname{sigE}$ and tryptase in about $31 \%$ and $48 \%$ of LAR patients, respectively. ${ }^{12,26} \mathrm{Al}-$ though, nasal sIgE is useful for detecting local sensitization after exposure to aeroallergens, it may not yield positive result in every case. This may be due to the specimen collection technique. Accordingly, a clinical criterion of NAPT is still required for the diagnosis of LAR.

The combination of limited knowledge about LAR and limited access to the necessary diagnostic equipment means that LAR is under diagnosed in many countries. It should be considered in CR patients with negative systemic atopy from SPT. Even though LAR and AR are different entities, they shared some similarities regarding management, such as allergen avoidance and medications, like oral antihistamine and intranasal steroids. ${ }^{12}$ Rondon, et al. conducted a randomized, double-blind, placebo controlled phase 2 clinical trial to investigate the efficacy and safety of subcutaneous immunotherapy (SCIT) with Dp in patients with LAR. ${ }^{14,15}$ The results revealed that SCIT produced significant improvement in the total symptom score and a reduction in the total medical score. It also improved nasal tolerance to NAPT with negative response in $50 \%$ of patients.

\section{Limitations}

This study has some mentionable limitations. First, PNIF requires patient cooperation to yield an accurate result; therefore, we included additional information (i.e., symptom score and MCA of ARM) for diagnosis. Second, nasal lavage technique may not be a suitable candidate technique for obtaining nasal secretion. An alternate technique may be placement of a nasal sponge in the nasal cavities after NAPT, since nasal sponge may be able to collect more concentrated secretion for in vitro analysis. Lastly, only Dp was used for NAPT, so the test was a monoallergen test. However, all included subjects were diagnosed CR with a history that strongly suggested allergy to house dust mite.

\section{Conclusion}

The prevalence of LAR to Dp in Thai adults with CR and negative skin prick test was $24.2 \%$. Most LAR patients had moderate-severe persistent severity. The clinical characteristics of LAR compared to NAR were quite similar to previous studies, except for sneezing, which was shown to be a significantly more dominant symptom in LAR than in NAR. Further studies in different allergens, the pathophysiology, molecular alteration, and treatment and follow-up in LAR should be performed in the future to enhance our understanding of this condition in Thai population.

\section{Acknowledgements}

The authors gratefully acknowledge Mrs. Jeerapa Kerdnoppakhun and Ms. Kanokporn Thalek for their assistance with manuscript preparation.

\section{Conflict of interest declaration}

All authors declare no personal or professional conflicts of interest, and no financial support from the companies that produce and/or distribute the drugs, devices, or materials described in this report.

\section{Funding disclosure}

This study was funded by a grant from the Faculty of Medicine Siriraj Hospital, Mahidol University, Bangkok, Thailand.

\section{References}

1. Wallace DV, Dykewicz MS, Bernstein DI, Blessing-Moore J, Cox L, Khan $\mathrm{DA}$, et al. The diagnosis and management of rhinitis: an updated practice parameter. J Allergy Clin Immunol. 2008;122(2 Suppl):S1-84.

2. Rosenfeld RM, Andes D, Bhattacharyya N, Cheung D, Eisenberg S, Ganiats TG, et al. Clinical practice guideline: adult sinusitis. Otolaryngol Head Neck Surg. 2007;137(3 Suppl):S1-31.

3. Seidman MD, Gurgel RK, Lin SY, Schwartz SR, Baroody FM, Bonner JR, et al. Clinical practice guideline: allergic rhinitis. Otolaryngol Head Neck Surg. 2015;152(1 Suppl):S1-43.

4. Bunnag C, Leurmarnkul W, Jareoncharsri P, Tunsuriyawong P, Assanasen P, Pawankar R. Quality of life assessment in Thai patients with allergic rhinoconjunctivitis using the SF-36 questionnaire (Thai version). Rhinology. 2005;43(2):99-103.

5. Bunnag C, Leurmarnkul W, Jareoncharsri P, Ungkanont K, Tunsuriyawong P, Kosrirukvongs P, et al. Development of a health-related quality of life questionnaire for Thai patients with rhinoconjunctivitis. Asian Pac J Allergy Immunol. 2004;22(2-3):69-79.

6. Meltzer EO, Blaiss MS, Naclerio RM, Stoloff SW, Derebery MJ, Nelson HS, et al. Burden of allergic rhinitis: allergies in America, Latin America, and Asia-Pacific adult surveys. Allergy Asthma Proc. 2012;33 Suppl 1:S113-41.

7. Vichyanond P, Suratannon C, Lertbunnaphong P, Jirapongsananuruk O, Visitsunthorn N. Clinical characteristics of children with non-allergic rhinitis vs with allergic rhinitis. Asian Pac J Allergy Immunol. 2010;28: 270-4.

8. Kanthawatana S, Maturim W, Fooanant S, Manorot M, Trakultivakorn M. Evaluation of threshold criteria for the nasal histamine challenge test in perennial allergic rhinitis. Asian Pac J Allergy Immunol. 1997;15(2):65-9.

9. Pawankar R, Bunnag C, Chen Y, Fukuda T, Kim YY, Le LT, et al. Allergic rhinitis and its impact on asthma update (ARIA 2008)--western and Asian-Pacific perspective. Asian Pac J Allergy Immunol. 2009;27(4):237-43.

10. Bunnag C, Jareoncharsri P, Tantilipikorn P, Vichyanond P, Pawankar R. Epidemiology and current status of allergic rhinitis and asthma in Thailand -- ARIA Asia-Pacific Workshop report. Asian Pac J Allergy Immunol. 2009;27(1):79-86.

11. Huggins KG, Brostoff J. Local production of specific IgE antibodies in allergic-rhinitis patients with negative skin tests. Lancet. 1975;2(7926): 148-50.

12. Rondon C, Romero JJ, Lopez S, Antunez C, Martin-Casanez E, Torres MJ, et al. Local IgE production and positive nasal provocation test in patients with persistent nonallergic rhinitis. J Allergy Clin Immunol. 2007;119(4): 899-905.

13. Platts-Mills TA. Local production of IgG, IgA and IgE antibodies in grass pollen hay fever. J Immunol. 1979;122(6):2218-25.

14. Rondon C, Blanca-Lopez N, Campo P, Mayorga C, Jurado-Escobar R, Torres MJ, et al. Specific immunotherapy in local allergic rhinitis: A randomized, double-blind placebo-controlled trial with Phleum pratense subcutaneous allergen immunotherapy. Allergy. 2018;73(4):905-15. 
APJAI

15. Rondon C, Blanca-Lopez N, Aranda A, Herrera R, Rodriguez-Bada JL, Canto G, et al. Local allergic rhinitis: allergen tolerance and immunologic changes after preseasonal immunotherapy with grass pollen. J Allergy Clin Immunol. 2011;127(4):1069-71.

16. Carney AS, Powe DG, Huskisson RS, Jones NS. Atypical nasal challenges in patients with idiopathic rhinitis: more evidence for the existence of allergy in the absence of atopy? Clin Exp Allergy. 2002;32(10):1436-40.

17. Tao XY, Ng CL, Chen D, Lin ZB, Wu SL, Liang MJ, et al. Clinical Characteristics and Allergen Sensitization Patterns of Patients with Local Allergic Rhinitis in Southern China. Int Arch Allergy Immunol. 2018;175(1-2):107-13.

18. Jung CG, Lee JH, Ban GY, Park HS, Shin YS. Prevalence and Clinical Characteristics of Local Allergic Rhinitis to House Dust Mites. Yonsei Med J. 2017;58(5):1047-50.

19. Buntarickpornpan P, Veskitkul J, Pacharn P, Visitsunthorn N, Vichyanond P, Tantilipikorn P, et al. The proportion of local allergic rhinitis to Dermatophagoides pteronyssinus in children. Pediatr Allergy Immunol. 2016;27(6):574-9.

20. Brozek J, Bousquet J, Baena-Cagnani C, Bonini S, Canonica G, Casale T, et al. Allergic Rhinitis and its Impact on Asthma (ARIA) guidelines: 2010 Revision. J Allergy Clin Immunol. 2010;126:466-76.
21. Rondon C, Campo P, Togias A, Fokkens WJ, Durham SR, Powe DG, et al. Local allergic rhinitis: concept, pathophysiology, and management. J Allergy Clin Immunol. 2012;129(6):1460-7.

22. Tantilipikorn P, Vichyanond P, Lacroix JS. Nasal provocation test: how to maximize its clinical use? Asian Pac J Allergy Immunol. 2011;28(4):225-31.

23. Tantilipikorn P, Jareoncharsri P, Voraprayoon S, Bunnag C, Clement PA. Acoustic rhinometry of Asian noses. Am J Rhinol. 2008;22(6):617-20.

24. Rondon C, Fernandez J, Lopez S, Campo P, Dona I, Torres MJ, et al. Nasal inflammatory mediators and specific IgE production after nasal challenge with grass pollen in local allergic rhinitis. J Allergy Clin Immunol. 2009;124(5):1005-11 e1.

25. Rondon C, Campo P, Galindo L, Blanca-Lopez N, Cassinello MS, Rodriguez-Bada JL, et al. Prevalence and clinical relevance of local allergic rhinitis. Allergy. 2012;67(10):1282-8.

26. Lopez S, Rondon C, Torres MJ, Campo P, Canto G, Fernandez R, et al. Immediate and dual response to nasal challenge with Dermatophagoides pteronyssinus in local allergic rhinitis. Clin Exp Allergy. 2010;40(7): $1007-14$ 